\title{
Lidocaine attenuates CFA-induced inflammatory pain in rats by regulating the MAPK/ERK/NF-кB signaling pathway
}

\author{
SHULI ZHANG ${ }^{1}$, YAN $\mathrm{LI}^{2}$ and YINGJUN TU ${ }^{3}$ \\ Departments of ${ }^{1}$ Pain Medicine and ${ }^{2}$ Gynecology, The First Affiliated Hospital of Xinjiang Medical University, Urumqi, \\ Xinjiang 830054; ${ }^{3}$ Department of Orthopaedics, Yili Friendship Hospital, Yining, Xinjiang 835000, P.R. China
}

Received May 19, 2020; Accepted October 8, 2020

DOI: $10.3892 /$ etm.2021.9643

\begin{abstract}
Lidocaine is a commonly used local anesthetic that also confers analgesic effects, resistance to hyperalgesia and anti-inflammatory properties. The present study aimed to explore the effects of lidocaine on complete Freund's adjuvant (CFA)-induced inflammatory pain. In the present study, rats were subcutaneously injected with CFA to investigate the molecular mechanisms associated with lidocaine in an inflammation-induced pain model. Firstly, CFA was subcutaneously injected into the paws of Sprague-Dawley rats, following which lidocaine or saline and the ERK agonist recombinant human epidermal growth factor (rh-EGF) were injected via the tail vein. Rat behavior was then assessed at 0 and $4 \mathrm{~h}$, 1, 4, 7 and 14 days after CFA treatment. Proinflammatory cytokine levels in the serum were measured using ELISA. Western blotting was performed to detect the protein levels of phosphorylated (p)-ERK1/2, ERK1/2 and NF- $\kappa$ B subunits p-p65 and p65. Reverse transcription-quantitative PCR was used to measure the mRNA expression of ERK1/2 and p65 in rat spinal cord tissues. The results showed that injection of CFA significantly reduced the mechanical withdrawal threshold, thermal withdrawal latency and the frequency of responses to cold stimulation in rats, whilst promoting tumor necrosis factor- $\alpha$, interleukin (IL)-1 $\beta$, IL- 6 levels in addition to ERK1/2, p65 protein phosphorylation. These effects were alleviated by lidocaine treatment. Furthermore, treatment with rh-EGF reversed the protective effects of lidocaine on inflammatory pain caused by CFA. In conclusion, lidocaine inhibits the inflammatory response and pain through the MAPK/ERK/NF- $\kappa$ B pathway in a rat model of pain induced by CFA.
\end{abstract}

Correspondence to: Dr Yingjun Tu, Department of Orthopaedics, Yili Friendship Hospital, 92 Stalin Street, Yining, Xinjiang 835000, P.R. China

E-mail: ty986789@163.com

Key words: lidocaine, inflammatory pain, MAPK/ERK/NF-кB pathway

\section{Introduction}

Pain is comprised of both physiological and pathological pain (1). Pathological pain can be further sub-divided into inflammatory, neuropathic and cancerous pain (2). Among them, as one of the common clinical symptoms, inflammatory pain affects $25-35 \%$ of adults in major European countries $(3,4)$. Current treatment strategies for pain combined with inflammation mainly consists of nonsteroidal anti-inflammatory drugs (NSAIDs) (5). However, long-term use of NSAIDs may cause gastrointestinal bleeding and other side effects, including acute myocardial infarction, heart failure and acute kidney injury (5). Therefore, it is necessary to discover novel safe and effective medication for inflammatory pain.

Lidocaine has been previously reported to improve tumor-free survival and overall survival by inhibiting the growth and metastasis of breast tumor cells $(6,7)$. In addition, lidocaine is a commonly used local anesthetic that also confers analgesic effects, resists hyperalgesia and anti-inflammatory properties $(8,9)$, which is conducive to postoperative analgesia and controlling the inflammatory response. Accumulating evidence has shown that lidocaine serves an important regulatory role in numerous aspects of the inflammatory response (10-12). It was also reported that the NF- $\mathrm{NB}$ signaling pathway is activated during inflammation (13), such that lidocaine has been found to exert anti-inflammatory effects by inhibiting the Toll-like receptor 4/NF- $\kappa$ B pathway (14).

Complete Freund's adjuvant (CFA) is a mixed oil that is often used to study the pathology and mechanism underlying inflammatory pain $(15,16)$. Nagakura et al (15) suggested that CFA can cause mechanical hyperalgesia in rats, where its inflammatory pain course can last for $>2$ weeks, making it a superior model for chronic pain research. In addition, Bai et al (16) previously established a rat model of inflammatory muscle pain by injecting CFA into the rat masseter muscle.

To the best of our knowledge, the effects of lidocaine on MAPK/ERK/NF- $\kappa \mathrm{B}$ signaling in CFA-induced chronic inflammation has not been reported previously. Therefore, in the present study, CFA was injected into Sprague-Dawley rats to establish a rat model of inflammatory pain, following which the role of lidocaine in inflammatory pain was assessed. 


\section{Materials and methods}

Animals. A total of 50 healthy male Sprague-Dawley rats (age, 4-6 weeks; weight, 150-200 g; Beijing Vital River Laboratory Animal Technology Co., Ltd.) were fed in a standard animal room with a 12-h light/dark cycle at a temperature of $22-25^{\circ} \mathrm{C}$ with 40-50\% humidity and free access to food and water. All protocols strictly followed the Institutional Animal Care and Use of Laboratory Animals by the National Institutes of Health (17). The experimental protocols were approved by the Animal Care and Use Committee of the First Affiliated Hospital of Xinjiang Medical University (Urumqi, China).

Establishment of a rat model and study groups. To establish a rat model of inflammatory pain, the plantar surface of the right hind paw of the rats was subcutaneously injected with $100 \mu \mathrm{l}$ CFA (Sigma-Aldrich; Merck KGaA). All rats were randomly assigned into five groups $(\mathrm{n}=10)$. In the control group, $100 \mu \mathrm{l}$ normal saline was injected into the tail vein of rats once a day for 4 days (18). In the CFA group, $100 \mu 1$ CFA was subcutaneously injected into rats. In the CFA + lidocaine group, rats were firstly treated with $100 \mu 1$ CFA, followed by injected with 1,3 or $5 \mathrm{mg} / \mathrm{kg}$ lidocaine (Sigma-Aldrich, Merck KGaA) into the tail vein $1 \mathrm{~h}$ later; lidocaine injections were performed once a day for 4 days. In the CFA + lidocaine + human epidermal growth factor (rh-EGF) group, rats were firstly injected with $100 \mu \mathrm{l} \mathrm{CFA}$, then $5 \mathrm{mg} / \mathrm{kg}$ lidocaine and $10 \mu \mathrm{g} / \mathrm{kg}$ rh-EGF were injected into the tail vein $1 \mathrm{~h}$ later. Lidocaine/rh-EGF injections were performed once a day for 4 days.

In total, 4 days after CFA injection, rats (4 rats from each group) were anesthetized with an intraperitoneal injection of $50 \mathrm{mg} / \mathrm{kg}$ pentobarbital and sacrificed by cervical dislocation. The peripheral blood and spinal cord tissues were subsequently harvested following euthanasia. Other rats (6 rats from each group) were subjected to behavioral tests (mechanical withdrawal threshold, thermal withdrawal latency and frequency responses to cold stimulation) at 0 and $4 \mathrm{~h}, 1,4,7$ or 14 days after CFA injection.

Behavioral tests. Rat behavioral tests assessed the mechanical withdrawal threshold (MWT), thermal withdrawal latency (TWL) and frequency responses to cold stimulation.

Mechanical hyperalgesia was evaluated using Von Frey monofilaments (Stoelting Co.). Von Frey monofilaments includes a set of 8 nylon filaments. The stimulus generated by the tip when it is bent is $\sim 0.5,1,2,4,6,8,10$ and $12 \mathrm{~g}$ (g, intensity unit). These eight stimuli were used to stimulate different positions on the plantar surface of the rats. The back paws of the rats were first put under increasing pressure lasting 5-6 sec, where the minimum force required to induce paw withdrawal was termed as the MWT.

For the thermal preference test, the response of each back paw was evaluated at intervals of 3 min after a radiant heater $\left(-3-65^{\circ} \mathrm{C}\right.$; cat. no. BME-410A; Institute of Biomedical Engineering, Chinese Academy of Medical Sciences) was placed under the plantar surface of the back paw. The TWL was defined as when the hind paws were removed from the thermal plate.

For the cold allodynia testing, a syringe was connected to a thin polyethylene tube to smear a drop of acetone to each back paw. A brisk paw withdrawal response was considered as a sign of cold hyperalgesia. The test was performed for three times and there were intervals of 5-10 min between each test.

All tests were performed after CFA injection for 0 and $4 \mathrm{~h}$, $1,4,7$ or 14 days.

ELISA. After 4 days of CFA injection, ELISA kits (Beyotime Institute of Technology) were used to measure TNF- $\alpha$ (cat. no. PT519), IL-1ß (cat. no. PI303) and IL-6 (cat. no. PI328) levels in rat serum according to the manufacturer's protocols. Antibodies were purchased from Beyotime Institute of Biotechnology. Each set of experiments was performed in triplicate.

Western blotting assay. The rats were euthanized after 4 days of CFA injection. RIPA buffer (Beyotime Institute of Technology) containing PMSF was used to extract total protein from the L4-L6 spinal cord tissues. Protein concentration was determined using a bicinchoninic acid protein assay kit (Pierce; Thermo Fisher Scientific, Inc.). Proteins ( $40 \mu \mathrm{g}$ per lane) were separated using $10 \%$ SDS-PAGE. Separated proteins were then transferred onto PVDF membranes. Subsequently, membranes were blocked with 5\% skimmed milk for $1 \mathrm{~h}$ at room temperature. Membranes were then incubated with primary antibodies: Erk1/2 (cat. no. 4695; dilution 1:1,000; Cell Signaling Technology, Inc.), phosphorylated (p)-Erk1/2 (cat. no. 4370; dilution 1:1,000; Cell Signaling Technology, Inc.), p65 (cat. no. 8242; dilution 1:1,000; Cell Signaling Technology, Inc.), p-p65 (cat. no. 3033; dilution 1:1,000; Cell Signaling Technology, Inc.) and GAPDH (cat. no. 5174; dilution 1:1,000; Cell Signaling Technology, Inc.) overnight at $4^{\circ} \mathrm{C}$. After three washes, membranes were incubated with horseradish peroxidase-conjugated secondary antibody (cat. no. 7074; dilution 1:2,000; Cell Signaling Technology, Inc.) for $1 \mathrm{~h}$ at $37^{\circ} \mathrm{C}$. Protein bands were visualized using Clarity Western ECL Substrate (Bio-Rad Laboratories, Inc.). Band densities were quantified using the Gel-Pro Analyzer densitometry software (version 6.3; Media Cybernetics, Inc.).

$R N A$ extraction and reverse transcription-quantitative PCR $(R T-q P C R)$. Spinal cord tissues were used to extract total RNA using an MagMAX ${ }^{\mathrm{TM}}$-96 Total RNA Isolation Kit according to manufacturer's protocols (Invitrogen; Thermo Fisher Scientific, Inc.). cDNA was synthesized using a PrimeScript ${ }^{\mathrm{TM}}$ RT reagent kit (Takara Bio, Inc.). The reaction conditions were as follows: $70^{\circ} \mathrm{C}$ for $5 \mathrm{~min}, 37^{\circ} \mathrm{C}$ for $5 \mathrm{~min}$ and $42^{\circ} \mathrm{C}$ for $60 \mathrm{~min}$. Power SYBR ${ }^{\mathrm{TM}}$-Green master mix (Applied Biosystems; Thermo Fisher Scientific, Inc.) was used to quantify mRNA expression. The following thermocycling conditions were used for the qPCR: Initial denaturation at $95^{\circ} \mathrm{C}$ for $5 \mathrm{~min}$; followed by 40 cycles of $15 \mathrm{sec}$ at $95^{\circ} \mathrm{C}, 1 \mathrm{~min}$ at $60^{\circ} \mathrm{C}$ and $30 \mathrm{sec}$ at $72^{\circ} \mathrm{C}$; and a final extension for $10 \mathrm{~min}$ at $72^{\circ} \mathrm{C}$. GAPDH was used as the internal control. The following primer sequences were used: GAPDH forward 5'-TTTGGTATCGTGGAAGGA CTC-3' and reverse, 5'-GTAGAGGCAGGGATGATGTTC T-3'; Erk forward 5'-GGCACCAACCATTGAGCAGA-3' and reverse, 5'-GATCATTGCTGAGGTGCTGTGTC-3'; p65 forward 5'-CGCGGATCCGCCACCATGGACGAACTG-3' and reverse, 5'-CCGCTCGAGTTAGGAGCTGATCTG-3'. Gene expression was calculated using the $2^{-\Delta \Delta C q}$ method (19). Experiments were performed in triplicate. 
A

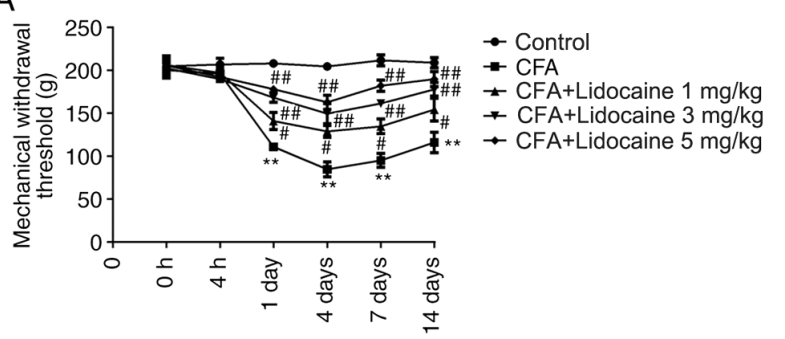

Time after CFA injection
B

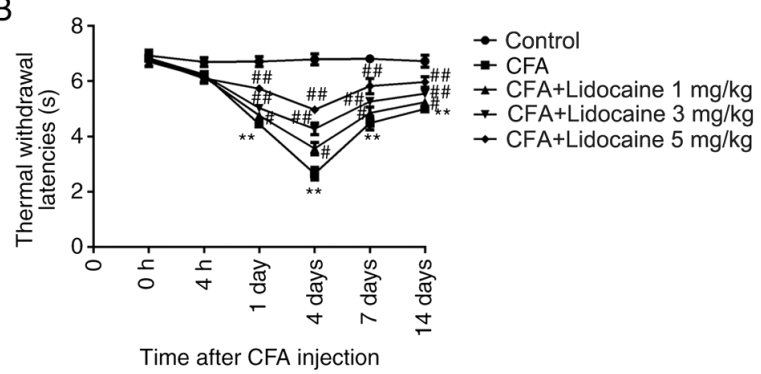

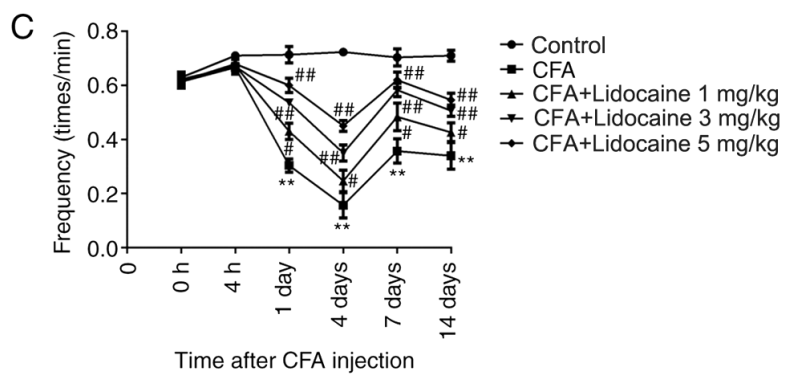

Figure 1. Effects of lidocaine on the behavior of CFA-treated rats. Rats were treated with lidocaine and CFA or CFA alone before rat behavior was assessed. (A) The effects of lidocaine on the MWT of CFA-treated rats. (B) The effects of lidocaine on the TWL of CFA-treated rats. (C) The effects of lidocaine on frequency responses to cold stimulation by CFA-treated rats. Data are presented as the mean \pm standard deviation. ${ }^{* *} \mathrm{P}<0.01$ vs. Control; ${ }^{*} \mathrm{P}<0.05$ and ${ }^{\# \#} \mathrm{P}<0.01$ vs. CFA. CFA, complete Freund's adjuvant; MWT, mechanical withdrawal threshold; TWL, thermal withdrawal latency.
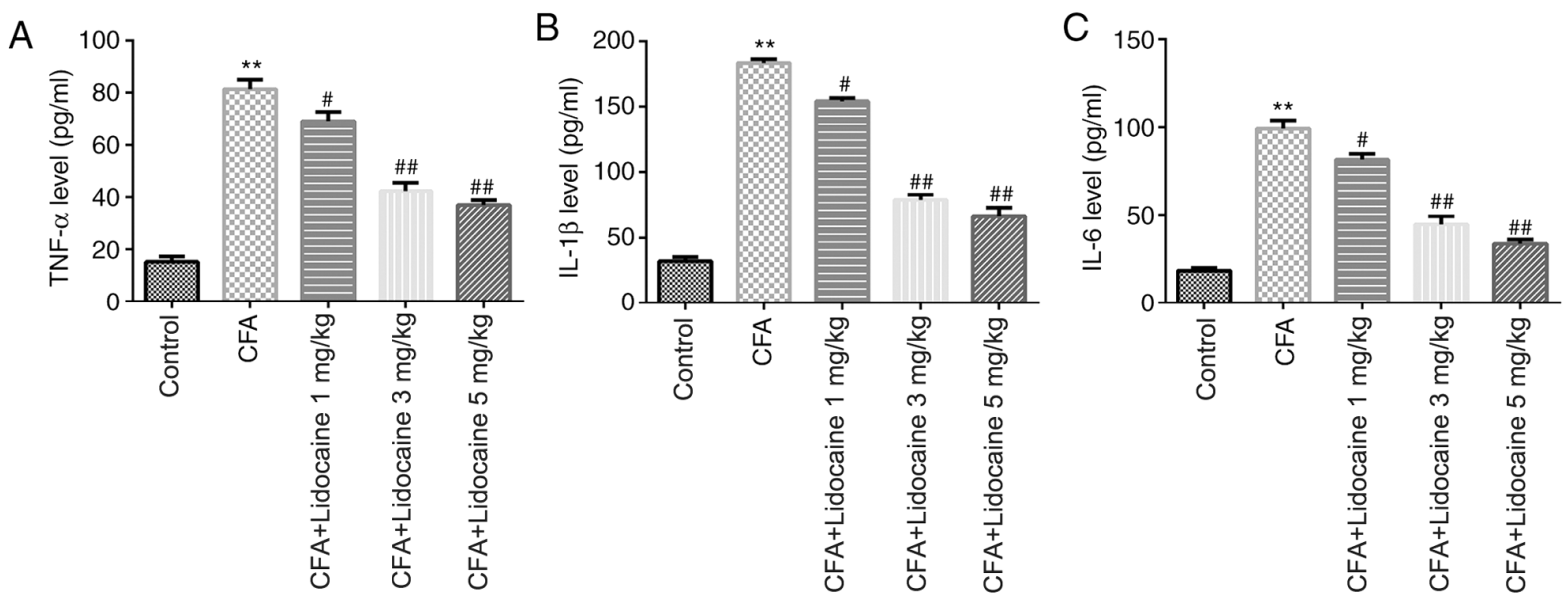

Figure 2. Effects of lidocaine on the levels of pro-inflammatory factors in the serum of CFA-treated rats. After 4 days of CFA treatment, the effects of lidocaine on the levels pro-inflammatory mediators were evaluated using ELISA. The effect of lidocaine on (A) TNF- $\alpha$, (B) IL-1 $\beta$ and (C) IL-6 levels in CFA-treated rats. Data are presented as the mean \pm standard deviation. ${ }^{* *} \mathrm{P}<0.01$ vs. Control; ${ }^{*} \mathrm{P}<0.05$ and ${ }^{\# /} \mathrm{P}<0.01$ vs. CFA. CFA, complete Freund's adjuvant; TNF, tumor necrosis factor; IL, interleukin.

Statistical analysis. Data are presented as the mean $\pm \mathrm{SD}$ from three independent experiments. Statistical analysis was performed using SPSS 18.0 (SPSS, Inc.). One-way ANOVA with Tukey's post hoc test was used for all comparisons. $\mathrm{P}<0.05$ was considered to indicate a statistically significant difference.

\section{Results}

Effects of lidocaine on the behavior of rats treated with CFA. Rats were first treated with either CFA alone or CFA + lidocaine. The TWL, MWT and frequency responses to cold stimulation were then assessed. The results showed that CFA significantly reduced TWL, MWT and the frequency responses to cold stimulation by rats after treatment for $1,4,7$ and 14 days. However, 1,3 and $5 \mathrm{mg} / \mathrm{kg}$ lidocaine significantly reversed these effects (Fig. 1A-C).

Lidocaine affects the levels of pro-inflammatory factors in the serum of CFA-induced rats and inhibits $M A P K / E R K / N F-\kappa B$ pathway activation. After 4 days of CFA treatment, ELISA was used to evaluate the levels pro-inflammatory mediators in the rat serum. The results showed that CFA injection significantly promoted TNF- $\alpha$, IL- $1 \beta$ and IL- 6 levels compared with those in the control group, all of which were significantly reversed by lidocaine $(1,3$ or $5 \mathrm{mg} / \mathrm{kg}$ ) (Fig. 2). 

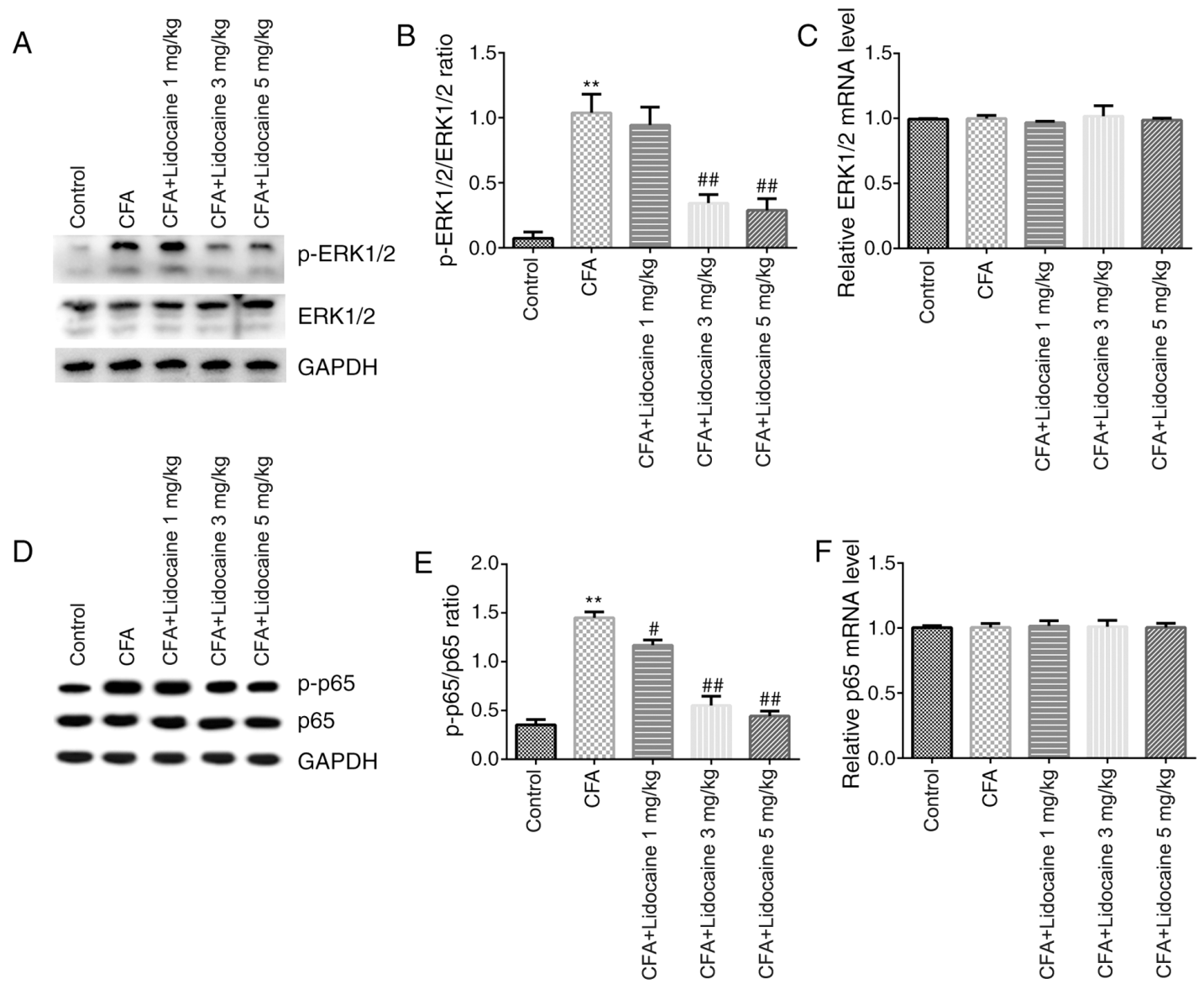

Figure 3. Effects of lidocaine on the MAPK/ERK/NF- $\mathrm{BB}$ signaling pathway in CFA-treated rats. To investigate the pathways in this rat model, after 4 days of CFA treatment, western blotting was used to measure ERK1/2 and p65 phosphorylation in rat spinal cord tissues. Reverse transcription-quantitative PCR was also used to detect the mRNA expression of ERK1/2 and p65. (A) The effect of lidocaine on p-ERK1/2 levels and the p-ERK1/2/ERK ratio in CFA-induced rats, (B) which was quantified. (C) The effect of lidocaine on ERK1/2 mRNA expression in CFA-treated rats. (D) The effect of lidocaine on p-p65 levels and the p-p65/p65 ratio in CFA-induced rats, (E) which was quantified. (F) The effect of lidocaine on p65 mRNA expression in CFA-treated rats. *"P $<0.01$ vs. Control; ${ }^{\#} \mathrm{P}<0.05$ and ${ }^{\# \#} \mathrm{P}<0.01$ vs. CFA. CFA, complete Freund's adjuvant; $\mathrm{p}$, phosphorylated.

Western blotting and RT-qPCR were then performed to assess the signaling pathways in rat spinal cord tissues. The results showed that protein levels of p-ERK1/2 (Fig. 3A) and p-p65 (Fig. 3D) in addition to the ratios of p-ERK1/2/ total-ERK1/2 (Fig. 3B) and p-p65/total-p65 (Fig. 3E) in the CFA group were significantly elevated. However, lidocaine ( 3 or $5 \mathrm{mg} / \mathrm{kg}$ ) significantly reversed the effects of CFA on ERK1/2 phosphorylation. Lidocaine $(1,3$ or $5 \mathrm{mg} / \mathrm{kg}$ ) significantly reversed the effects of CFA on p65 phosphorylation. Furthermore, the mRNA expression of ERK1/2 and p65 did not significantly differ after CFA treatment (Fig. $3 \mathrm{C}$ and F).

Lidocaine reduces inflammatory pain following CFA injection by affecting the MAPK/ERK-NF- $B$ pathway. Compared with those after lidocaine treatment alone, in the presence of CFA, lidocaine + rh-EGF significantly reduced TWL, MWT and frequency responses to cold stimulation at days $1,4,7$ and 14 (Fig. 4A-C) and significantly increased TNF- $\alpha$, IL-1 $\beta$ and IL- 6 levels in the rat serum at 4 days after CFA treatment (Fig. 4D-F).

In addition, in the presence of CFA, lidocaine + rh-EGF treatment significantly increased the levels of p-ERK $1 / 2$ and the p-ERK1/2/total-ERK1/2 ratio when compared with those after lidocaine treatment alone (Fig. 5A and B). There were no significant differences in ERK1/2 mRNA expression between the lidocaine and lidocaine + rh-EGF groups (Fig. 5C). In addition, lidocaine + rh-EGF significantly increased the levels of p-p65 and the p-p65/total-p65 ratio compared with those after lidocaine treatment alone (Fig. 5D and E). There were no significant differences in p65 mRNA expression between either treatment groups (Fig. 5F). The results suggested that lidocaine relieved inflammatory pain caused by CFA, which was reversed by rh-EGF in rats, suggesting that lidocaine relieved inflammatory pain caused by CFA via inhibiting the MAPK/ERK/NF-кB pathway.

\section{Discussion}

Lidocaine is a commonly used anesthetic in clinical practice (20). In recent years, studies have previously shown that lidocaine exerts beneficial pharmacological effects for treating inflammatory reactions, such that it has been successfully applied to a variety of inflammatory diseases, including acute thyroiditis and inflammatory pain $(21,22)$. The present study found that lidocaine reduced inflammatory pain in rats induced by CFA based on the results of behavioral tests. 


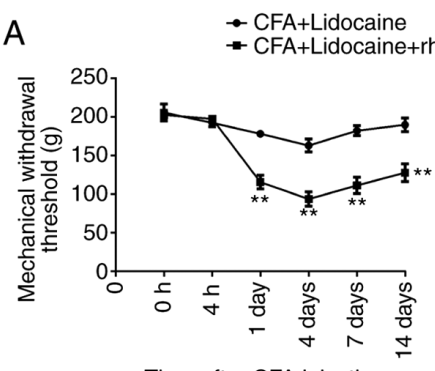

Time after CFA injection

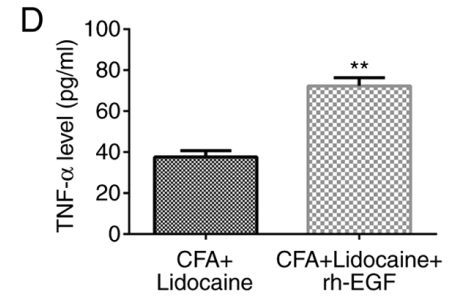

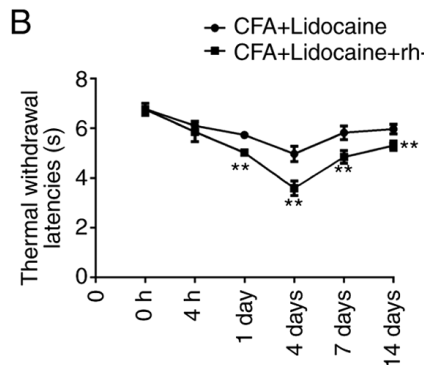

Time after CFA injection

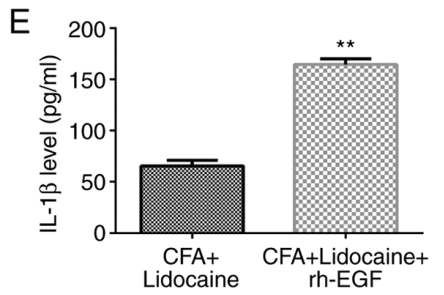

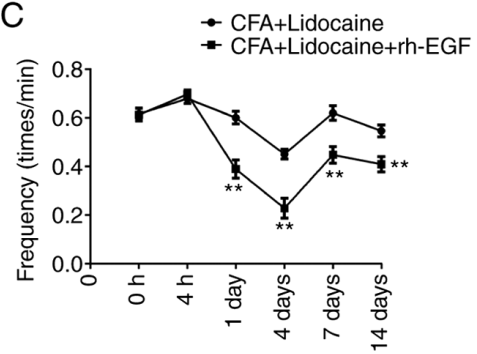

Time after CFA injection

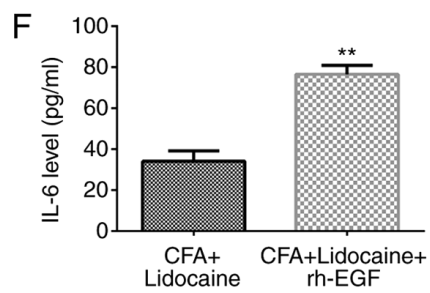

Figure 4. Protective effects of lidocaine on the behavior CFA-treated rats is reversed by rh-EGF. CFA treated rats were treated with the ERK agonist rh-EGF based on aforementioned studies. The effect of lidocaine and rh-EGF on the (A) mechanical withdrawal threshold, (B) thermal withdrawal latency and (C) frequency responses to cold stimulation by CFA-induced rats were analyzed at 0 and $4 \mathrm{~h}, 1,4,7$ or 14 days after CFA treatment. The effect of lidocaine and rh-EGF on the serum levels of (D) TNF- $\alpha,(\mathrm{E})$ IL- $1 \beta$ and (F) IL- 6 in CFA-treated rats were determined at 4 days after CFA treatment. ${ }^{* *}$ P $<0.01$ vs. CFA + lidocaine. CFA, complete Freund's adjuvant; rh-EGF, recombinant human epidermal growth factor; TNF, tumor necrosis factor; IL, interleukin.

A

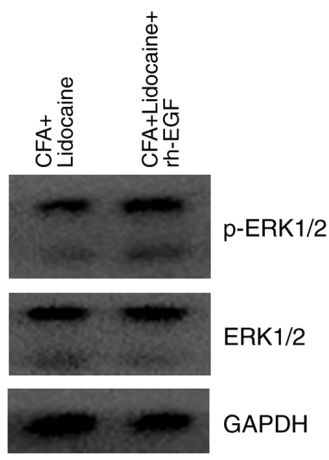

D

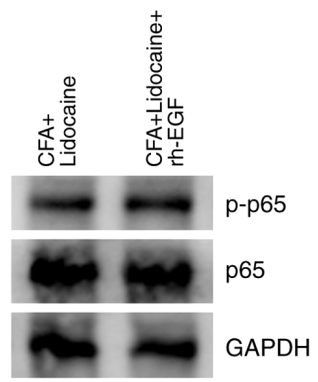

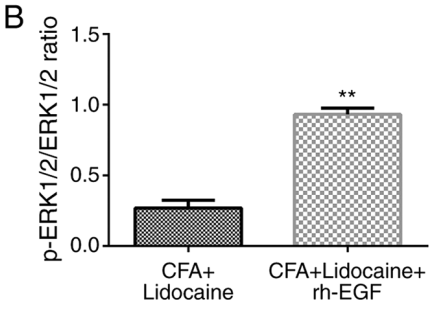

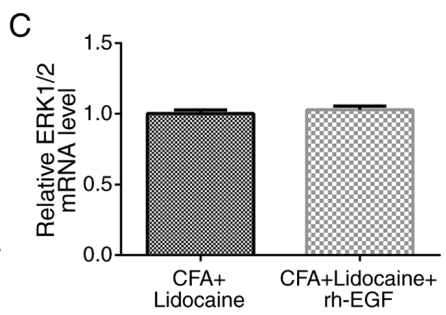

E
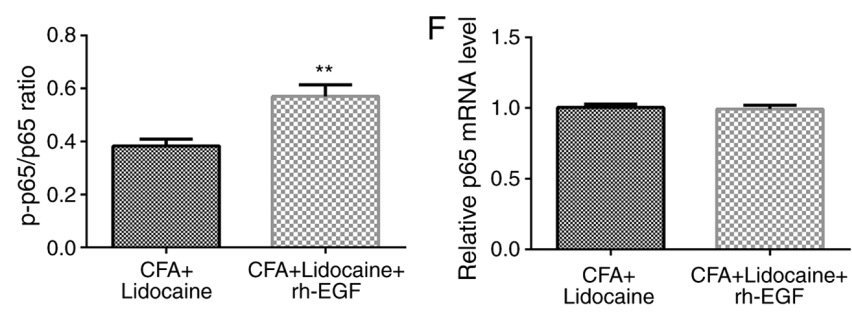

Figure 5. Effects of lidocaine on the MAPK/ERK/NF- $\kappa \mathrm{B}$ pathway in CFA-induced rats is reversed by rh-EGF. Effects of lidocaine on the MAPK/ERK/NF- $\mathrm{B}$ pathway in CFA-induced rats were studied at 4 days after CFA treatment. (A) The effect of lidocaine and rh-EGF on p-ERK1/2 levels and the p-ERK1/2/ERK ratio of CFA-induced rats, (B) which was quantified. (C) The effect of lidocaine and rh-EGF on ERK1/2 mRNA expression in CFA-treated rats. (D) The effect of lidocaine and rh-EGF on p-p65 levels and the p-p65/p65 ratio in CFA-treated rats, (E) which was quantified. (F) The effect of lidocaine and rh-EGF on p65 mRNA expression in CFA-treated rats. Data are presented as the mean \pm standard deviation. ${ }^{* *} \mathrm{P}<0.01$ vs. CFA + lidocaine. CFA, complete Freund's adjuvant; p-, phosphorylated; rh-EGF, recombinant human epidermal growth factor.

Lidocaine is known to have a short half-life when given intravenously $(23,24)$. Studies have previously investigated the potential use of lidocaine for many headache disorders, primarily via injection or infusion (24). A previous study reported that intravenous systemic lidocaine infusion is beneficial for patients with pain uncontrolled by opioid medications and can improve pain scores whilst reducing the need for opioid treatment (25). Systemic administration of lidocaine is antinociceptive in both chronic and acute pain states, especially in acute postoperative and chronic neuropathic pain (26). In the present study, 1, 3 and $5 \mathrm{mg} / \mathrm{kg}$ lidocaine were used to treat CFA-induced inflammatory pain in rats via tail vein injection based on a previous study (27). 
TNF- $\alpha$ is a widely bioactive peptide substance that is secreted by activated macrophages, endothelial cells, neutrophils, B lymphocytes, monocytes, dendritic cells, neurons and astrocytes (28). TNF- $\alpha$ activates neutrophils and macrophages, promoting the production of IL-1 $\beta$, IL- 6 and other Th2 cytokines, such as IL- 4 and IL-5 to serve a key role in the inflammatory response (29). IL-1 $\beta$ and IL-6 levels has been used to objectively reflect the degree of inflammation and are usually highly expressed during inflammatory reactions (30). It has also been reported to be positively correlated with the severity of the inflammatory pain (31-34). In the present study, the ELISA results were consistent with a previous study (35), which showed that CFA injection significantly increased inflammation-associated factors TNF- $\alpha$, IL-1 $\beta$ and IL-6. The present results also showed that lidocaine reversed the inflammatory effects of CFA in rats.

MAPKs are located in eukaryotic cytoplasm that serve as key signal transductors to transduce extracellular signals to intracellular responses in eukaryotic cells. MAPKs can connect key regulatory factors and cell surface receptors, mediating a variety of biological responses to serve key roles in physiological and pathological processes $(36,37)$. ERK is a major member of the MAPK pathway family. There are two types of ERK: ERK1 and ERK2. After phosphorylation, p-ERK1/2 enters the nucleus and regulates the expression of transcription factors, including cyclin D and cyclin E, to promote cell proliferation (38). NF- $\kappa \mathrm{B}$ is a protein with transcriptional activation function, with the p65/p50 complex being the main form (39). Inactivated $\mathrm{NF}-\kappa \mathrm{B}$ is located in the cytoplasm, but when cells are stimulated by cytokines, $\mathrm{NF}-\kappa \mathrm{B}$ then translocates into the nucleus to activate gene transcription (40). Studies have previously shown that $\mathrm{NF}-\kappa \mathrm{B}$ can promote the expression of inflammatory factors IL-1, TNF- $\alpha$ and IL- 6 during hepatic fibrosis $(41,42)$. In the present study, western blotting results showed that injection of CFA increased ERK1/2 and p65 protein phosphorylation but lidocaine treatment reversed this. However, no significant differences in total ERK1/2 and p65 mRNA expression was observed by either CFA and/or lidocaine treatment.

Next, to further confirm that lidocaine inhibits the MAPK/ERK/NF- $\kappa \mathrm{B}$ pathway to relieve inflammatory pain caused by CFA, CFA-induced rats were treated with rh-EGF (an ERK agonist) based on data from the present study. The results suggested that compared with those after lidocaine treatment alone, lidocaine + rh-EGF treatment significantly reduced TWL, MWT and frequency responses to cold stimulation whilst significantly increasing TNF- $\alpha$, IL-1 $\beta$ and IL-6 levels. In addition, the levels of ERK $1 / 2$ and p65 phosphorylation and the ratios of p-ERK1/2/total-ERK1/2 and p-p65/total-p65 were significantly increased, whilst the mRNA expression of ERK1/2 and p65 were not significantly different between these two groups. These findings indicated that the effects of lidocaine on CFA-induced rats were significantly reversed by rh-EGF. Since rh-EGF is an ERK agonist (43), data from the present study suggest that rh-EGF + lidocaine significantly reduced TWL, MWT and frequency responses to cold stimulation but significantly increased TNF- $\alpha$, IL- $1 \beta$ and IL- 6 levels through activating the ERK pathway.

In summary, these results indicated that lidocaine inhibited the activation of the MAPK/ERK-NF- $\mathrm{B}$ pathway, to inhibit the inflammatory response and alleviate inflammatory pain caused by CFA. This study provides more theoretical basis for the use of lidocaine for the clinical treatment of inflammatory pain.

\section{Acknowledgements}

Not applicable.

\section{Funding}

No funding was received.

\section{Availability of data and materials}

The datasets used and/or analyzed during the present study are available from the corresponding author on reasonable request.

\section{Authors' contributions}

SZ designed the current study, in addition to performing all experiments and analyzing the data. YL contributed to performing ELISA, western blot assay, reverse transcription-quantitative PCR and analyzing the data. YT contributed to establishing the rat model conduction and behavioral tests and analyzing the data. All authors read and approved the final manuscript.

\section{Ethics approval and consent to participate}

All protocols strictly followed the Institutional Animal Care and Use of Laboratory Animals by the National Institutes of Health. The experimental protocols were approved by the Animal Care and Use Committee of the First Affiliated Hospital of Xinjiang Medical University (approval no. IACUC-20200113-01; Urumqi, China).

\section{Patient consent for publication}

Not applicable.

\section{Competing interests}

The authors declare that they have no competing interests.

\section{References}

1. Swieboda P, Filip R, Prystupa A and Drozd M: Assessment of pain: Types, mechanism and treatment. Ann Agric Environ Med 1: 2-7, 2013.

2. Grace PM, Hutchinson MR, Maier SF and Watkins LR: Pathological pain and the neuroimmune interface. Nat Rev Immunol 14: 217-231, 2014

3. Fang JQ, Du JY, Liang Y and Fang JF: Intervention of electroacupuncture on spinal p38 mapk/atf-2/vr-1 pathway in treating inflammatory pain induced by CFA in rats. Mol Pain 9: 13, 2013.

4. Del Giorno R, Frumento P, Varrassi G, Paladini A and Coaccioli S: Assessment of chronic pain and access to pain therapy: A cross-sectional population-based study. J Pain Res 10: 2577-2584, 2017

5. Zhang B, He XL, Ding Y and Du GH: Gaultherin, a natural salicylate derivative from gaultheria yunnanensis: Towards a better non-steroidal anti-inflammatory drug. Eur J Pharmacol 530: 166-171, 2006. 
6. Chamaraux-Tran TN and Tobias P: The amide local anesthetic lidocaine in cancer surgery-potential antimetastatic effects and preservation of immune cell function? A narrative review. Front Med (Lausanne) 4: 235, 2017.

7. Johnson MZ, Crowley PD, Foley AG, Xue C, Connolly C, Gallagher HC and Buggy DJ: Effect of perioperative lidocaine on metastasis after sevoflurane or ketamine-xylazine anaesthesia for breast tumour resection in a murine model. $\mathrm{Br} \mathrm{J}$ Anaesth 121: 76-85, 2018.

8. Lauretti GR: Mechanisms of analgesia of intravenous lidocaine. Rev Bras Anestesiol 58: 280-286, 2008 (In En, Portuguese).

9. Kawamata M, Takahashi T, Kozuka Y, Nawa Y, Nishikawa K, Narimatsu E, Watanabe $\mathrm{H}$ and Namiki A: Experimental incision-induced pain in human skin: Effects of systemic lidocaine on flare formation and hyperalgesia. Pain 100: 77-89, 2002

10. Wang L, Wang M, Li S, Wu H, Shen Q, Zhang S, Fang L and Liu R: Nebulized lidocaine ameliorates allergic airway inflammation via downregulation of TLR2. Mol Immunol 97: 94-100, 2018.

11. Chiu KM, Lu CW, Lee MY, Wang MJ, Lin TY and Wang SJ: Neuroprotective and anti-inflammatory effects of lidocaine in kainic acid-injected rats. Neuroreport 27: 501-507, 2016.

12. van der Wal SEI, van den Heuvel SAS, Radema SA, van Berkum BFM, Vaneker M, Steegers MAH, Scheffer GJ and Vissers KCP: The in vitro mechanisms and in vivo efficacy of intravenous lidocaine on the neuroinflammatory response in acute and chronic pain. Eur J Pain 20: 655-674, 2016.

13. Liu LM, Liang DY, Ye CG, Tu WJ and Zhu T: The UII/UT system mediates upregulation of proinflammatory cytokines through p38MAPK and NF- $\kappa$ B pathways in LPS stimulated Kupffer cells. PLoS One 10: e0121383, 2015.

14. Wang HL, Xing YQ, Xu YX, Rong F, Lei WF and Zhang WH: The protective effect of lidocaine on septic rats via the inhibition of high mobility group box 1 expression and NF- $\kappa \mathrm{B}$ activation. Mediators Inflamm 2013: 570370, 2013

15. Nagakura Y, Okada M, Kohara A, Kiso T, Toya T, Iwai A, Wanibuchi $\mathrm{F}$ and Yamaguchi T: Allodynia and hyperalgesia in adjuvant-induced arthritic rats: Time course of progression and efficacy of analgesics. J Pharmacol Exp Ther 306: 490-497, 2003.

16. Bai G, Ambalavanar R, Wei D and Dessem D: Downregulation of selective microRNAs in trigeminal ganglion neurons following inflammatory muscle pain. Mol Pain 3: 15, 2007.

17. Bayne K: Revised guide for the care and use of laboratory animals available. American physiological society. Physiologist 39: 208-211, 1996

18. Tan SS, Liu H, Wang YZ and Zhu SS: The molecular mechanisms associated with the effects of propofol in a rat model of pain due to inflammation following injection with complete freund's adjuvant. Med Sci Monit 25: 10190-10197, 2019.

19. Livak KJ and Schmittgen TD: Analysis of relative gene expression data using real-time quantitative PCR and the 2(-Delta Delta C(T)) method. Methods 25: 402-408, 2001

20. Klein JA and Jeske DR: Estimated maximal safe dosages of tumescent lidocaine. Anesth Analg 122: 1350-1359, 2016.

21. Yang X, Yang LX, Wu J, Guo ML, Zhang Y and Ma SG: Treatment of lidocaine on subacute thyroiditis via restraining inflammatory factor expression and inhibiting pyroptosis pathway. J Cell Biochem: Apr 8, 2019 (Epub ahead of print).

22. Leon-Constantin MM, Alexa-Stratulat T, Luca A, Tamba BI, Trandafir LM, Harabagiu V and Cojocaru E: The morphofunctional impact of topical lidocaine formulation in inflammatory pain-experimental study. Rom J Morphol Embryol 60: 869-874, 2019.

23. Soto G, Naranjo González M and Calero F: Intravenous lidocaine infusion. Rev Esp Anestesiol Reanim 65: 269-274, 2018 (In En, Spanish).

24. Berk T and Silberstein SD: The use and method of action of intravenous lidocaine and its metabolite in headache disorders. Headache 58: 783-789, 2018.

25. Reeves DJ and Foster AE: Continuous intravenous lidocaine infusion for the management of pain uncontrolled by opioid medications. J Pain Palliat Care Pharmacother 31: 198-203, 2017.
26. Hermanns H, Hollmann MW, Stevens MF, Lirk P, Brandenburger T, Piegeler T and Werdehausen R: Molecular mechanisms of action of systemic lidocaine in acute and chronic pain: A narrative review. Br J Anaesth 123: 335-349, 2019.

27. Chen LJ, Ding YB, Ma PL, Jiang SH, Li KZ, Li AZ, Li MC, Shi CX, Du J and Zhou HD: The protective effect of lidocaine on lipopolysaccharide-induced acute lung injury in rats through NF- $\kappa \mathrm{B}$ and p38 MAPK signaling pathway and excessive inflammatory responses. Eur Rev Med Pharmacol Sci 22: 2099-2108, 2018.

28. Caminero A, Comabella M and Montalban X: Tumor necrosis factor alpha (TNF- $\alpha$ ), anti-TNF- $\alpha$ and demyelination revisited: An ongoing story. J Neuroimmunol 234: 1-6, 2011

29. Luo Y, Pang Z, Zhu Q, Cai X, Yin Y, Wang M, Zhu J, Chen J, Zeng $\mathrm{K}$, Zhang $\mathrm{C}$ and Zhang J: Locally instilled tumor necrosis factor- $\alpha$ antisense oligonucleotide inhibits allergic inflammation via the induction of Tregs. J Gene Med 14: 374-383, 2012.

30. Slaats J, Ten Oever J, van de Veerdonk FL and Netea MG: IL-1 $\beta /$ IL-6/CRP and IL-18/ferritin: Distinct inflammatory programs in infections. PLoS Pathog 12: e1005973, 2016.

31. Dai T, Shi KQ, Chen G, Shen YM and Pan T: Malvidin attenuates pain and inflammation in rats with osteoarthritis by suppressing NF- $\kappa$ B signaling pathway. Inflamm Res 66: 1075-1084, 2017.

32. Dokumaci A: Performing pain and inflammation: Rendering the invisible visible. AMA J Ethics 19: 834-838, 2017.

33. Barrientos RM, Hein AM, Frank MG, Watkins LR and Maier SF: Intracisternal interleukin-1 receptor antagonist prevents postoperative cognitive decline and neuroinflammatory response in aged rats. J Neurosci 32: 14641-14648, 2012.

34. Wan Y, Xu J, Ma D, Zeng Y, Cibelli M and Maze M: Postoperative impairment of cognitive function in rats: A possible role for cytokine-mediated inflammation in the hippocampus. Anesthesiology 106: 436-443, 2007.

35. Yuan Y, Zhang Y, He X and Fan S: Protective effects of sinomenine on CFA-induced inflammatory pain in rats. Med Sci Monit 24: 2018-2024, 2018.

36. Brown MD and Sacks DB: Compartmentalised MAPK pathways. Handb Exp Pharmacol 186: 205-235, 2008.

37. Aouadi M, Binetruy B, Caron L, Le Marchand-Brustel Y and Bost F: Role of MAPKs in development and differentiation: Lessons from knockout mice. Biochimie 88: 1091-1098, 2006.

38. Zhong W, Shen WF, Ning BF, Hu PF, Lin Y, Yue HY, Yin C, Hou JL, Chen YX, Zhang JP, et al: Inhibition of extracellular signal-regulated kinase 1 by adenovirus mediated small interfering RNA attenuates hepatic fibrosis in rats. Hepatology 50: 1524-1536, 2009.

39. Giridharan S and Srinivasan M: Mechanisms of NF- $\kappa$ B p65 and strategies for therapeutic manipulation. J Inflamm Res 11: 407-419, 2018.

40. Sunami Y,LeithäuserF, GulS, Fiedler K, Güldiken N, Espenlaub S, Holzmann KH, Hipp N, Sindrilaru A, Luedde T, et al: Hepatic activation of IKK/NFKB signaling induces liver fibrosis via macrophage-mediated chronic inflammation. Hepatology 56: $1117-1128,2012$.

41. Chen X, Liu C, Lu Y, Yang Z, Lv Z, Xu Q, Pan Q and Lu L: Paeoniflorin regulates macrophage activation in dimethylnitrosamine-induced liver fibrosis in rats. BMC Complement Altern Med 12: 254, 2012

42. Gao Y, Xi B, Li J, Li Z, Xu J, Zhong M, Xu Q, Lian Y, Wei R, Wang L, et al: Scoparone alleviates hepatic fibrosis by inhibiting the TLR-4/NF- $\kappa$ B pathway. J Cell Physiol: Oct 8, 2020 (Epub ahead of print)

43. Liu CH, Hua N, Fu X, Pan YL, Li B and Li XD: Metformin regulates atrial SK2 and SK3 expression through inhibiting the PKC/ERK signaling pathway in type 2 diabetic rats. BMC Cardiovasc Disord 18: 236, 2018.

This work is licensed under a Creative Commons Attribution-NonCommercial-NoDerivatives 4.0 International (CC BY-NC-ND 4.0) License. 